Les plasmas naturels ou de laboratoire sont le lieu privilégié de structurations de l'émission lumineuse, s'apparentant à des phénomènes de morphogénèse et traduisant des effets collectifs de la dynamique des particules chargées.

Le travail que nous exposons ici porte sur l'auto-organisation de filaments dans des décharges à barrières diélectriques ; il se base sur l'utilisation conjointe d'un modèle 2D prédictif décrivant la physique du transport des particules chargées, et d'un dispositif expérimental quasi monodimensionnel permettant des comparaisons qualitatives directes avec le modèle. Nous dévoilons pour

la première fois l'existence d'une décharge optiquement " invisible», qui se produit autour de chaque filament et qui est essentielle à la génération et au mouvement des structures organisées.

\title{
Phénomènes d'auto-organisation dans les plasmas froids hors équilibre
}

Jean-Pierre Boeuf (jpb@laplace.univ-tlse.fr), Benoît Bernecker, Thierry Callegari,

Stéphane Blanco et Richard Fournier

LAPLACE (Laboratoire plasma et conversion d'énergie), UMR CNRS 5213,

Université Toulouse 3 - Paul Sabatier, 118 route de Narbonne, 31062 Toulouse Cedex 9

\section{Plasmas froids et structures organisées}

Les plasmas, systèmes complexes et non linéaires, sont le siège d'une multitude de phénomènes d'instabilités et d'auto-organisation. Un exemple bien connu est celui des plasmas magnétisés de fusion, dans lesquels l'existence de gradients de densité et de température considérables entre le cœur et le bord est à l'origine d'instabilités conduisant à une auto-organisation magnétique du plasma [1,2]. Des phénomènes de turbulence et de structurations cohérentes existent également dans les plasmas magnétisés naturels, comme celui de la magnétosphère [3].

D'autres types de plasmas, très différents des précédents et beaucoup plus accessibles, les plasmas froids hors équilibre (cf. encadré 1) offrent également un ensemble fascinant d'exemples de structures auto-organisées. On peut citer la "boule plasma ", bien connue du grand public (fig. 1).

L'exemple que nous choisissons ici pour illustrer les phénomènes d'auto-organisation dans les plasmas froids est celui des " décharges à barrières diélectriques "
(DBD), dans lesquelles des filaments de plasma lumineux se forment dans un espace gazeux entre deux diélectriques recouvrant des électrodes soumises à une tension sinusoïdale de basse fréquence (fig. 2). Les filaments qui se développent perpendiculairement aux diélectriques peuvent être stables mais, dans la majorité des cas, ils adoptent un comportement dynamique. Ces plasmas sont utilisés industriellement pour le traitement de surfaces. L'idéal pour le procédé serait que le plasma soit parfaitement homogène, ce qui n'est pratiquement jamais le cas, comme le montre la figure 2 (p. 18). L'intérêt, purement scientifique, de comprendre les mécanismes d'auto-organisation conduisant aux structures de la figure $2 \mathrm{~b}$ est donc associé à une exigence industrielle.

Pour un physicien du non linéaire, les plasmas froids de DBD de la figure 2 sont certainement une des manifestations les plus spectaculaires des phénomènes de morphogénèse en milieu dissipatif, dont A. Turing avait prévu mathématiquement l'existence [5] et que I. Prigogine a théorisé dans le contexte de la thermodynamique hors équilibre [6]. De plus, un des grands

\section{Les plasmas froids hors équilibre}

Les plasmas froids hors équilibre thermodynamique, beaucoup moins énergétiques que les plasmas de fusion, sont caractérisés par une température de gaz basse (voisine de l'ambiante) et des températures électroniques de quelques eV (dizaines de milliers de K). Contrairement au cas des plasmas de fusion ou de la magnétosphère, le degré d'ionisation (proportion d'atomes ou de molécules ionisés) est faible dans les plasmas hors équilibre (typiquement inférieur à $10^{-3}$ ) ; les collisions entre particules chargées et neutres y jouent un rôle essentiel, et les instabilités qui se forment sont souvent liées aux collisions et au couplage entre transport des particules chargées et champ électrique.

Ces plasmas, générés par des champs électriques éventuellement en présence de champs magnétiques, sont utilisés dans nombre d'applications industrielles ou domestiques: ozonizeurs, traitement de surfaces, dépôt de couches minces et gravure pour la microélectronique, lampes, écrans à plasma, etc. 


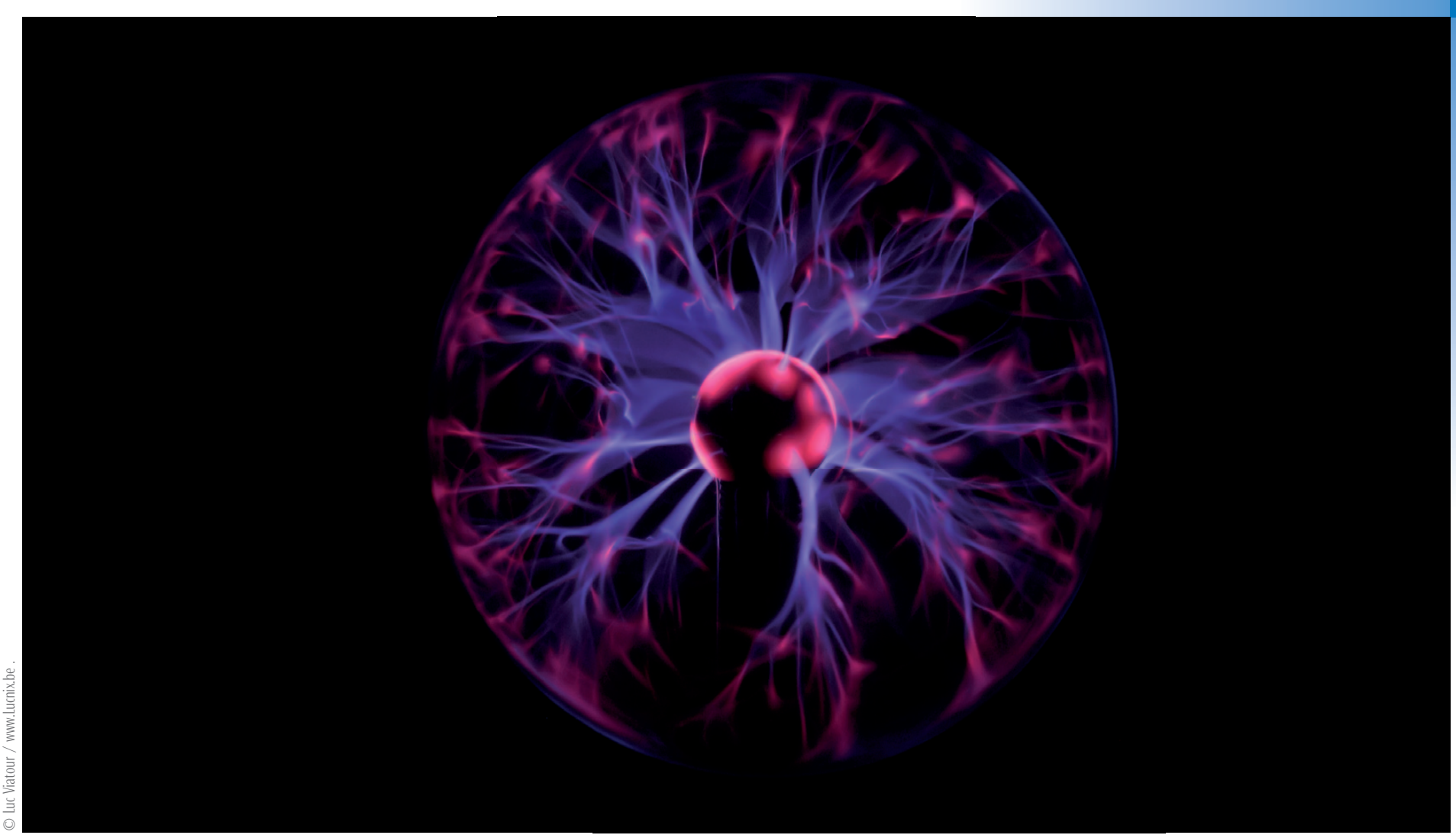

1. Auto-organisation de filaments dans une «boule plasma ». Des filaments auto-organisés de plasma en mouvement transportent le courant entre une électrode (petite boule pleine au centre) soumise à une haute tension de basse fréquence et la masse environnante, séparées capacitivement par une enveloppe diélectrique transparente (mélange de gaz sous basse pression).

avantages des DBD est leur facilité de mise en œuvre en laboratoire et la simplicité avec laquelle la diversité des structures est explorée en agissant sur les conditions opératoires (intensité et forme des tensions appliquées, pression du gaz, etc.) Ces plasmas de décharge permettent notamment l'étude expérimentale reproductible des plus intrigants parmi les phénomènes précurseurs de l'auto-organisation [7, 8], tels que la génération, l'annihilation et les « collisions " de filaments, la rotation de paires de filaments, etc.
Pourtant, malgré l'intérêt qu'ils suscitent, ces phénomènes restent largement incompris et sont sujets à controverse. En effet, les modèles de transport sont fiables [9] (et prédisent quantitativement les structures observées), mais trop complexes pour une étude de mécanismes à l'aide des outils de la physique non linéaire, alors que les modèles réacto-diffusifs, plus adaptés, manquent de fondement théorique (cf. encadré 2).

Le travail rapporté ici apparaît comme la première proposition aboutie d'un jeu de mécanismes permettant d'interpréter à la fois l'émergence des principales structures observées et leur sensibilité aux conditions opératoires.

Cette proposition a vu le jour grâce à la conception d'un dispositif expérimental quasi unidimensionnel inspiré de celui de la réf. [10], avec de meilleures résolutions temporelles, mais surtout une géométrie permettant de doubler l'analyse expérimentale d'une analyse théorique appuyée sur un modèle "fluidique " 2D (cf. encadré 2). Le dispositif est présenté sur la figure 3.

\section{Modèles de plasma de DBD}

On peut distinguer deux principaux types de modèles susceptibles d'aider à la compréhension de l'auto-organisation dans les DBD.

Les modèles semi-empiriques de type réaction-diffusion décrivent l'émergence spontanée d'une structure spatio-temporelle sous les effets conjoints d'un apport extérieur d'énergie et des interactions à l'œuvre entre les éléments du système considéré. Les structures auto-organisées résultent de la compétition entre une amplification locale (" activation »), et un mécanisme d'inhibition plus global. Dans les DBD, les activateurs peuvent être la tension aux bornes du gaz et la densité volumique de particules chargées, et l'inhibiteur les charges accumulées en surface des diélectriques, qui diffusent plus vite dans la direction transverse au courant de décharge, que la décharge elle-même.

Le second type de modèle, de type "fluidique », s'appuie sur les mécanismes physiques de base du problème. Il est décrit par un ensemble d'équations : (i) des équations de continuité, avec un terme source fortement non linéaire qui représente la création d'électrons et d'ions ; (ii) des équations de transport de quantité de mouvement, où le flux d'électrons ou d'ions est la somme d'un terme de dérive proportionnel au champ électrique et d'un terme de diffusion proportionnel au gradient de densité ; (iii) couplées à l'équation de Poisson reliant le gradient de champ électrique à la somme des charges de volume et de surface, avec les conditions aux limites appropriées (tension appliquée).

Nous avons opté pour les modèles du second type, comportant la physique essentielle du problème dans nos conditions expérimentales. Le système d'équations correspondant peut être résolu numériquement à deux ou trois dimensions. 

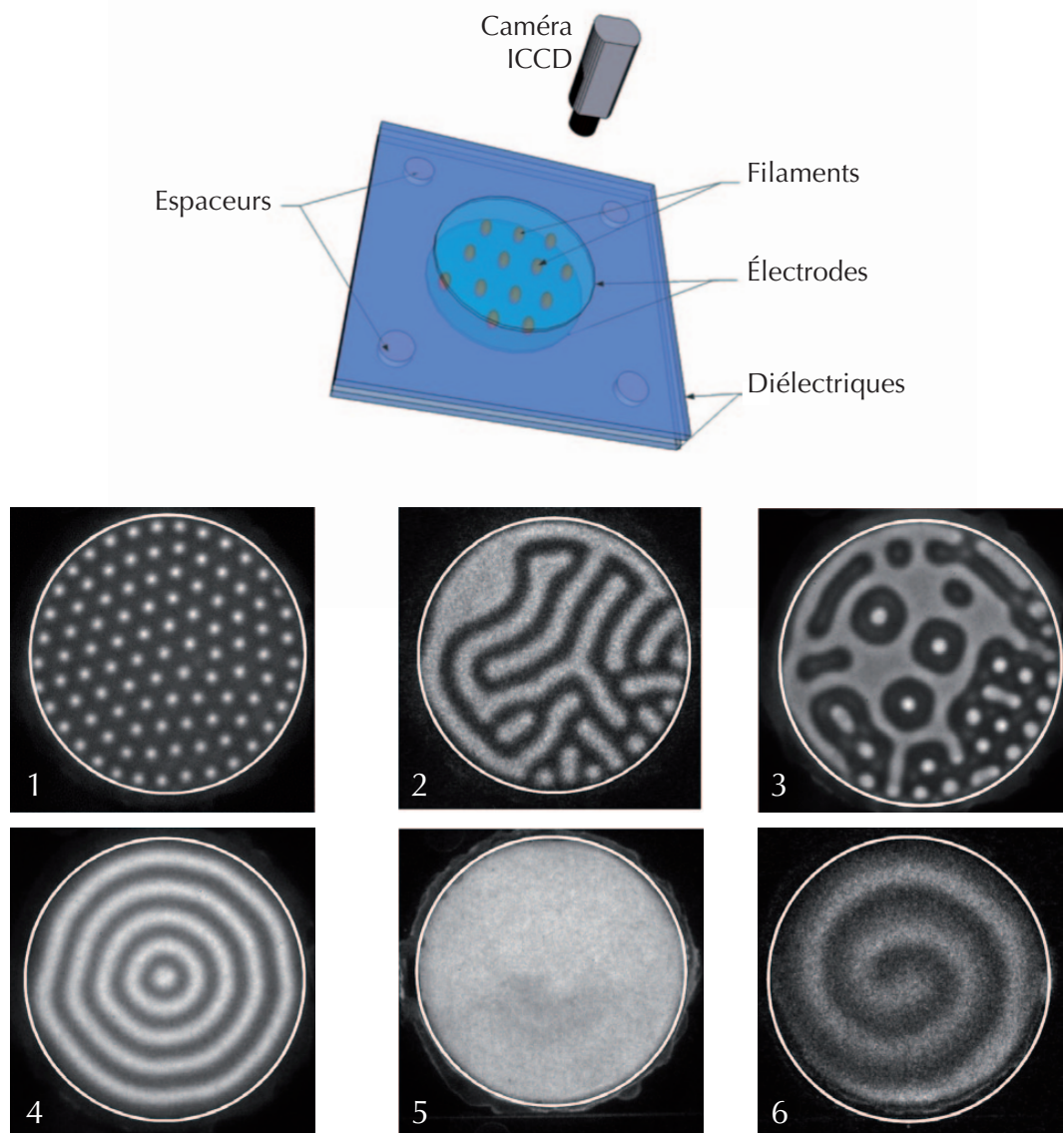

2. Décharge à barrière diélectrique dans un dispositif bidimensionnel.

(a) Schéma du dispositif expérimental ; les structures filamentaires sont observées à travers une électrode conductrice transparente (couche d'oxyde d'indium dopé à l'étain (IT0), par exemple), de diamètre $\phi=51 \mathrm{~mm}$.

(b) Exemples de structures organisées observées : structure hexagonale de filaments (1), en bandes (2), hybride (3), en cible (4), d’apparence homogène (5), en spirale (généralement en rotation) (6).
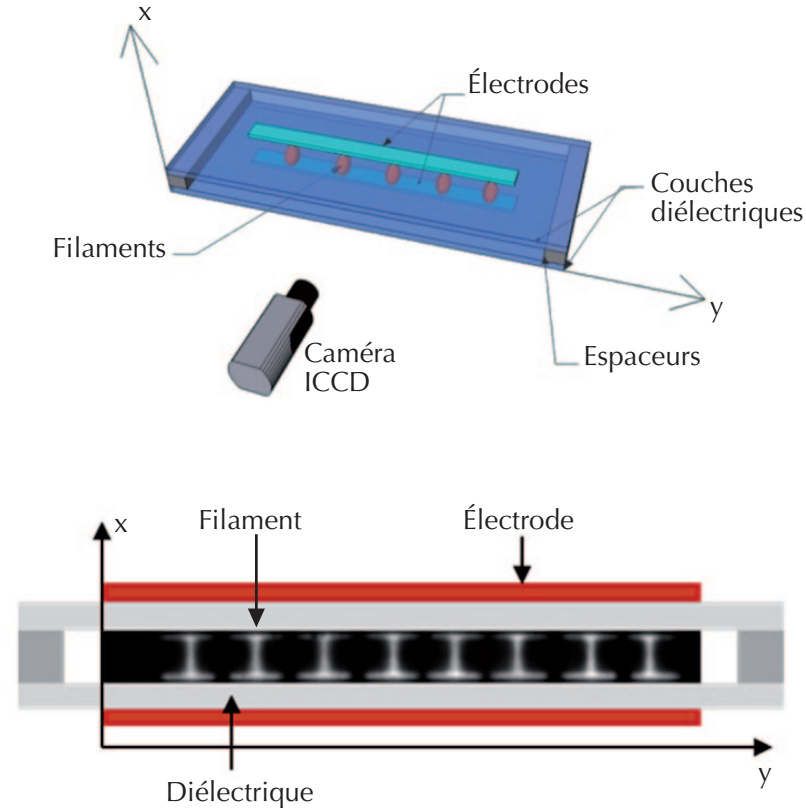

3. Décharge à barrière diélectrique dans un dispositif quasi monodimensionnel.

(a) Schéma du dispositif expérimental. La distance interdiélectrique est de $2 \mathrm{~mm}$, et la longueur de l'électrode est de $70 \mathrm{~mm}$ pour une largeur de $5 \mathrm{~mm}$. Les structures filamentaires sont observées perpendiculairement à l'axe des filaments.

(b) Exemple d'une structure filamentaire quasi périodique, classiquement observée dans des gaz rares.
$>>$

\section{Décharges à barrière diélectrique (DBD) et filaments de plasma}

Dans une décharge à barrière diélectrique, un plasma froid est généré dans l'espace gazeux entre deux couches diélectriques recouvrant deux électrodes planes (figs $2 \mathrm{a}$ et 3a). L'épaisseur des couches diélectriques et de l'espace gazeux est autour du mm, le gaz est souvent un gaz rare à une pression de l'ordre de $10^{4} \mathrm{~Pa}$. Au-delà d'une tension seuil entre les électrodes, le gaz passe de l'état isolant à l'état conducteur (plasma). La présence des diélectriques limite capacitivement le courant et impose donc l'utilisation de tensions variables au cours du temps. Très souvent elles sont de forme sinusoïdale, à des fréquences de quelques centaines de $\mathrm{Hz}$ à la centaine de $\mathrm{kHz}$, pour des amplitudes allant de quelques centaines de volts à plusieurs kilovolts. Un plasma se forme alors à chaque alternance de la tension et disparait en raison de la chute de tension dans le gaz due à la charge des diélectriques par le courant traversant le plasma. Dans les conditions indiquées ci-dessus, le plasma qui se forme est très rarement homogène, mais se présente sous forme de filaments perpendiculaires aux électrodes et qui s'organisent suivant des structures plus ou moins complexes (fig. 2b).

À des tensions voisines du seuil, on observe des filaments isolés, fixes ou se déplaçant le long de la surface, dont le comportement s'apparente à celui de solitons dissipatifs. Les filaments de plasma sont créés et disparaissent à chaque alternance de la tension, mais à ces fréquences, l'œil ne perçoit que la présence continue d'un filament.

Les filaments se reforment, à chaque alternance, au même endroit (ou "presque " au même endroit) car les charges électriques qu'ils déposent sur les couches diélectriques créent un effet mémoire. En effet, la tension due aux charges de surface s'oppose à la tension appliquée au cours d'une alternance, ce qui conduit à l'extinction du filament, mais s'ajoute à la tension appliquée à l'alternance suivante (changement de signe de la tension appliquée), ce qui permet de rallumer le filament au même endroit. Ces phénomènes sont illustrés sur la figure 4 dans le cas d'une décharge monodimensionnelle, où le plasma ne peut s'étaler que dans une seule direction. 


\section{Activation-inhibition, génération de filaments et formation d'une structure organisée}

Pour comprendre l'auto-organisation dans les décharges à barrière diélectrique, on peut étudier de façon détaillée la genèse d'une structure à l'aide de modèles fluidiques de plasmas (cf. encadré 2, p. 17).

Ces modèles 2D (une dimension axiale de la décharge, indispensable pour décrire les filaments, plus une dimension transverse, correspondant aux phénomènes de structuration) permettent de simuler l'évolution des décharges dans le plan de la figure $3 b$, qui présente le dispositif quasimonodimensionnel. Dans cette géométrie, les deux électrodes sont des bandes parallèles. Les décharges filamentaires s'organisent donc dans la direction parallèle aux électrodes (i.e. direction y de la figure 3 ), et on peut les observer, à l'aide de caméras rapides, dans le plan de la figure $3 \mathrm{~b}$. Les observations sont alors directement comparables aux résultats des calculs $2 \mathrm{D}$ présentés ci-dessous. La figure 5 , notamment, montre comment une non-uniformité initiale peut évoluer pour former un filament, puis une structure périodique de filaments synchrones (une seule décharge de courant à chaque alternance de la tension appliquée) dans la direction y de la figure 3.

Le phénomène d'activation est lié à la formation du plasma dès que la tension vue par l'espace gazeux dépasse une valeur seuil. La formation d'un filament inhibe le démarrage d'une décharge dans son voisinage immédiat (comme expliqué dans les légendes des figures $4 \mathrm{~b}$ et $5 \mathrm{~b}$ ). On voit également, à l'instant $t_{3}$ de la figure $5 b$, qu'une décharge électrique de plus faible intensité se forme dans la zone (3) au-delà de la région d'inhibition (zone (2)), parce que d'une part, dans cette région, la tension vue par le gaz est suffisante pour initier une décharge, et d'autre part des particules chargées ont eu le temps de diffuser du filament central vers cette région. Une des conditions nécessaires à la formation de structures dans un système de type réactiondiffusion est que l'inhibiteur (les charges accumulées en surface des diélectriques dans notre cas) diffuse plus vite que l'activateur (tension aux bornes du gaz et charges en volume). C'est ce qui est observé entre les instants $t_{1}, t_{2}$, et $t_{3}$ de la figure $5 b$.
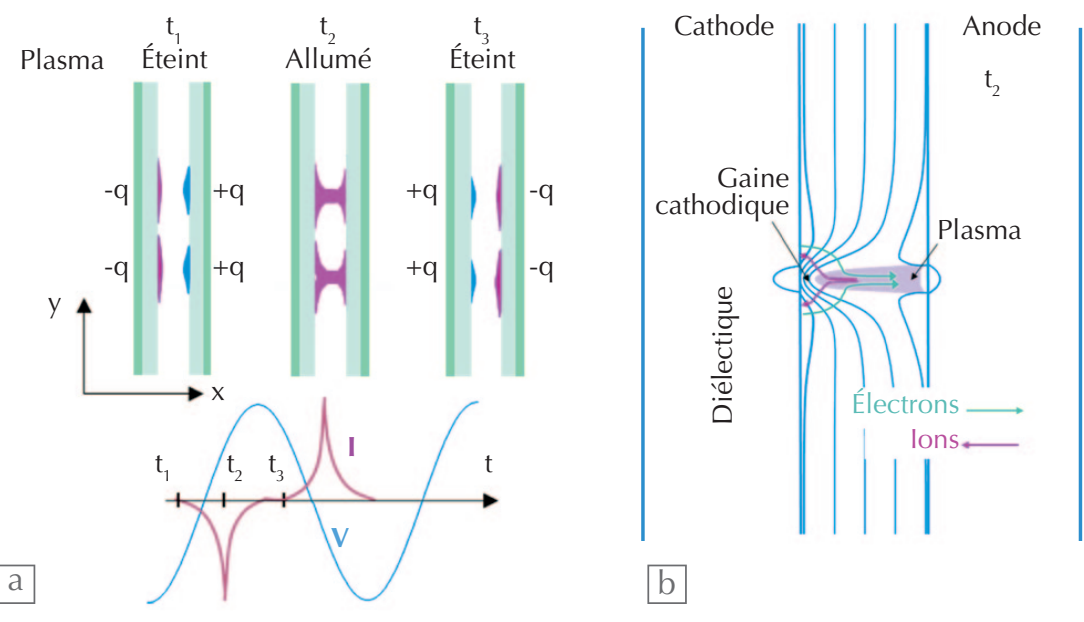

4. Formation et disparition d'un filament de plasma dans une DBD soumise à une tension V de forme sinusoïdale.

(a) L'état homogène du plasma n'est pas stable : dès qu'une perturbation se forme, par exemple dans la densité de charge déposée sur la surface des diélectriques (état à l'instant t), la décharge de l'alternance de tension suivante démarrera plus vite à l'endroit où les charges de surface sont plus grandes; ceci entraîne la formation d'un filament de plasma (état à l'instant $\mathrm{t}_{2}$ ), qui charge le diélectrique en sens inverse et disparaît en raison de la charge des diélectriques (impulsion de courant de la courbe rouge I(t)). En effet, la tension vue par le gaz à cet endroit, somme de la tension appliquée (courbe bleue $V(t)$ ) et de la tension mémoire due aux charges déposées, est devenue trop faible pour entretenir la décharge.

(b) La déformation des lignes d'isopotentiel électrique (courbes bleues), due à la présence du filament et à la charge de surface entraîne un étalement des ions (flèches rouges) le long de la surface, tandis que les électrons émis par la surface en raison du bombardement ionique (flèches vertes) se multiplient dans le champ électrique élevé de la gaine cathodique (zone proche du diélectrique, côté cathode) et convergent vers l'intérieur du filament (le filament, conducteur, tend à être équipotentiel et la plus grande partie de la tension est redistribuée dans la gaine cathodique). Cet étalement des ions entraîne une chute rapide de la tension vue par le gaz au voisinage du filament, et donc inhibe la formation d'un autre filament en son voisinage immédiat.

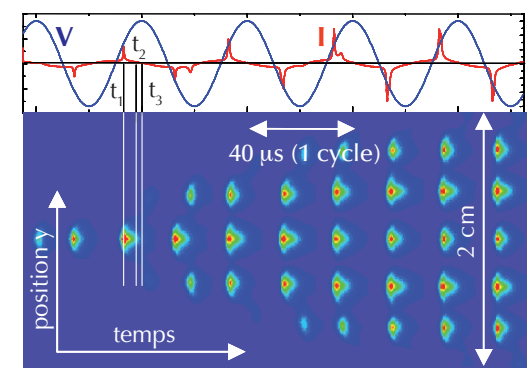

a

\section{a.}

5. Formation d'une structure organisée de décharges filamentaires synchrones dans une DBD quasi monodimensionnelle.

(a) Évolution temporelle, sur environ 5 périodes de la tension appliquée, de la distribution longitudinale (selon y) de la densité d'ions intégrée axialement (selon x) dans du néon à $25 \mathrm{kHz}$ et $600 \mathrm{~V}$, pour une distance interdiélectriques et une épaisseur des diélectriques de $2 \mathrm{~mm}$. La tension appliquée (courbe bleue) et le courant calculé (courbe rouge) sont tracés au-dessus de la figure. On suppose, à $t=0$, une distribution gaussienne de densité d'électrons et d'ions, centrée en $y=1 \mathrm{~cm}$. Celle-ci conduit, en quelques demi-périodes, à la formation d'un filament, puis, de proche en proche, à la génération de nouveaux filaments qui finissent par former une structure périodique synchrone.

(b) Distribution de la densité d'ions dans l'espace interdiélectriques à trois instants d'une demi-période, indiqués sur la figure a (valeurs maximales à $\mathrm{t}_{1}: 2,410^{11} \mathrm{~cm}^{-3}, \mathrm{t}_{2}: 2,310^{10} \mathrm{~cm}^{-3}, \mathrm{t}_{3}: 810^{9} \mathrm{~cm}^{-3}$ ). Sont indiquées également sur cette figure les lignes équipotentielles (en blanc). La densité électronique est égale à la densité ionique dans la région quasi équipotentielle (plasma, zone (1)), tandis que la zone de transition (appelée gaine) entre le plasma et la surface de gauche est une couche ionique qui assure la continuité du courant, et dans laquelle le champ est élevé en raison de la faible conductivité ionique. L'étalement des ions le long de cette surface crée une zone d'inhibition autour du filament (zone (2)), tandis qu'une décharge électrique à faible courant se forme dans la zone (3), au-delà de la zone d'inhibition. 

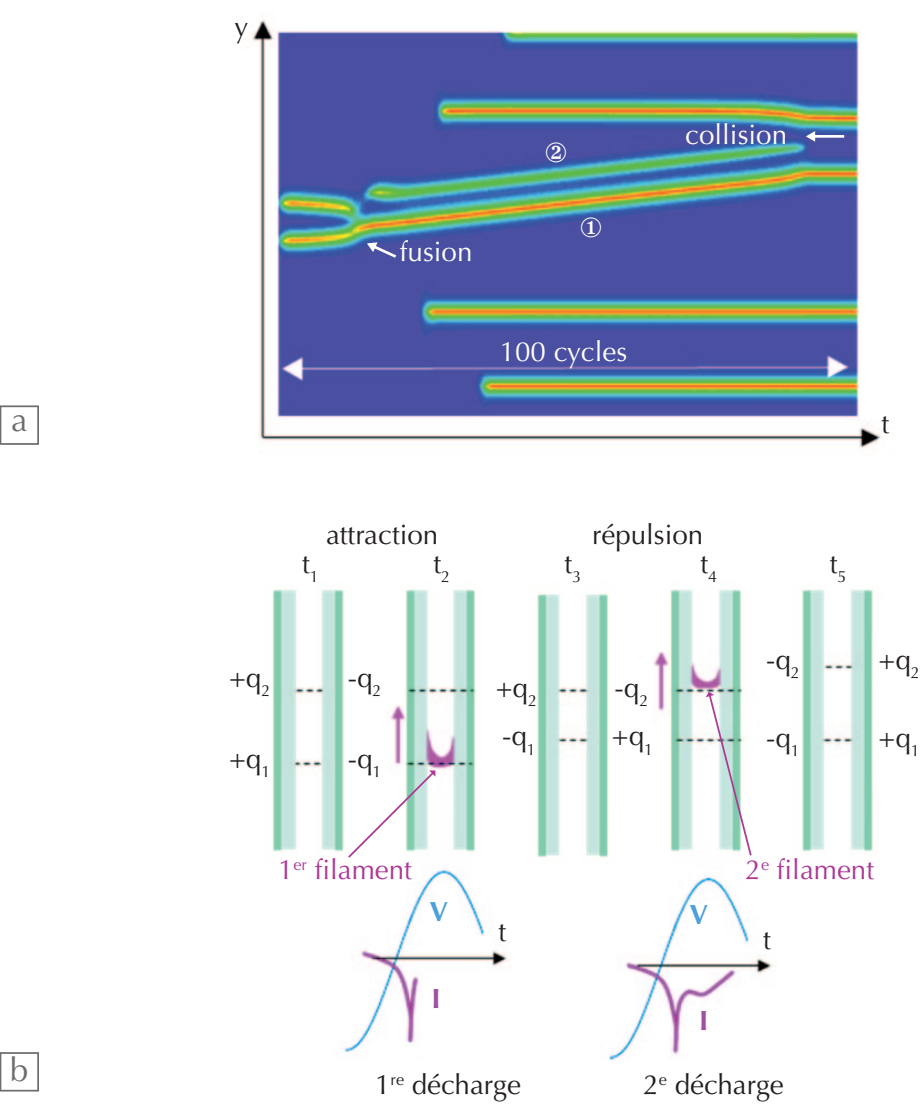

6. Évolution temporelle de filaments en interaction dans une DBD quasi monodimensionnelle, calculée dans un modèle fluidique $2 \mathrm{D}$.

(a) Évolution temporelle, sur 100 périodes de la tension appliquée, de la distribution longitudinale de la densité d'ions intégrée axialement et moyennée sur une période. Remarquer le déplacement de la paire asymétrique de filaments, (1) et (2).

(b) Description schématique du mécanisme d’attraction-répulsion qui explique le déplacement de la paire de filaments, vu sur la figure a : une dissymétrie est créé après la fusion des filaments centraux (flèche blanche sur la figure a), donnant naissance à un « gros » et à un « petit » filament, laissant des charges mémoires $\pm q_{1}$ et $\pm q_{2}$ respectivement, sur les surfaces diélectriques (instant $\mathrm{t}_{1}$ ). En début d'un cycle (instant $\mathrm{t}_{2}$ ), la décharge filamentaire se forme d'abord en vis-à-vis de la charge $q_{1}\left(\operatorname{car} q_{1}>q_{2}\right)$. La dissymétrie électrique associée à la présence de la charge $q_{2}$ sur la surface entraîne un léger déplacement (flèche violette) du filament et des charges qu’il dépose sur la surface, vers $q_{2}$. Après l'extinction du premier filament, les charges de surface $+q_{1}$ et $-q_{1}$ sont inversées (instant $t_{3}$ ). Dès que la tension appliquée (qui continue à monter) est suffisante, une décharge se produit en vis-à-vis de la charge de surface $q_{2}$ (instant $t_{4}$ ). La présence des charges $q_{1}$ inversées repousse la seconde décharge vers le haut. Le déplacement de la paire est donc associé à un phénomène d'attraction-répulsion.
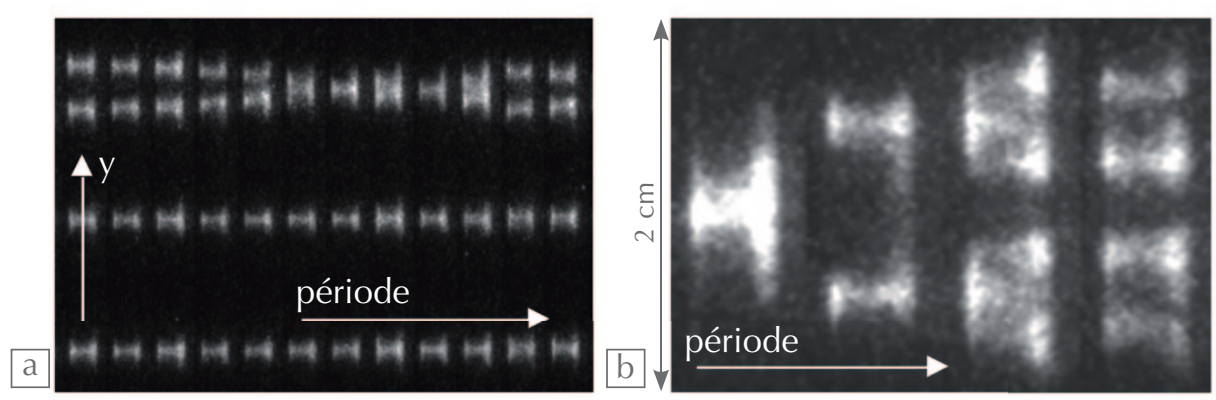

7. Exemples de visualisations de décharges successives, obtenues avec une caméra rapide dans le plan XY de la figure 3. (a) Images de 12 décharges successives : chaque image est intégrée sur la durée de la période de la tension appliquée. On observe deux filaments stables et deux filaments qui fusionnent puis se redivisent. (b) Image agrandie, montrant deux divisions successives sur quatre périodes.

\section{$>>$}

L'intensité de la seconde décharge qui se produit sur les côtés d'un filament (et qui apparait avant la formation de nouveaux filaments sur la figure 5 , zone (3)), et la quantité de charges qu'elle transfere vers le diélectrique dépendent essentiellement de l'amplitude et de la fréquence de la tension appliquée. Ces deux paramètres conditionnent donc complètement l'évolution ultérieure de ces décharges secondaires. Dans le cas de la figure 5, ces dernières deviennent elles-mêmes des filaments après quelques alternances, pour in fine former une structure organisée périodique.

\section{Annihilation/fusion et dynamique de filaments}

Si la tension appliquée est proche du seuil d'allumage du plasma, on voit souvent des filaments isolés susceptibles d'interagir. La figure 6a montre un exemple de calcul dans ces conditions.

On démarre le calcul avec deux nonuniformités de densités initiales proches l'une de l'autre, à une tension légèrement supérieure à la tension seuil. Ces deux nonuniformités deviennent, en quelques cycles, des filaments. La distance entre ces derniers est cependant inférieure à la zone d'inhibition, et ils finissent par fusionner pour n'en former qu'un seul. Cependant, la charge totale résultant de cette fusion est supérieure à celle nécessaire pour l'entretien d'un filament dans ces conditions, et on en voit un second (noté (2)) de moindre intensité se former au-dessus du premier (noté (1). On assiste alors au déplacement le long de la surface de cette paire asymétrique de filaments, jusqu'à ce que d'autres se forment et que la paire de filaments entre en collision avec l'un d'entre eux, conduisant ainsi à la disparition de la décharge de plus faible intensité. Le déplacement de la paire de filaments est associé à un mécanisme d'attraction-répulsion décrit sur la figure $6 \mathrm{~b}$. La rupture de symétrie qui permet le déplacement est ici due à la fusion des deux filaments initiaux.

Des phénomènes de fusion, d'annihilation et de déplacement de filaments, comparables à ceux prédits par les modèles, peuvent être visualisés expérimentalement à l'aide du dispositif monodimensionnel présenté sur la figure 3. La figure 7 montre quelques images obtenues avec une caméra rapide. 


\section{Conclusion}

Il n'existe pas d'analyse théorique permettant de comprendre globalement la génération, dans les plasmas de DBD, de structures variées, stationnaires ou dynamiques, telles que celles de la figure $2 b$, parfois semblables à des formes d'auto-organisation classiques caractéristiques de systèmes réacto-diffusifs. L'utilisation conjointe d'imagerie rapide et de modèles fluidiques permet cependant d'identifier les mécanismes élémentaires à l'origine de ces phénomènes.

Plusieurs approches peuvent être envisagées pour arriver à une compréhension plus globale. La première, et la plus satisfaisante d'un point de vue théorique, serait de construire, à partir d'équations fluidiques physiques, un modèle de la dynamique non linéaire responsable de l'auto-organisation. La deuxième utiliserait des modèles de type réacto-diffusif, appuyés sur les observations et sur des résultats de simulations numériques. Enfin, une troisième approche serait de partir des observations et simulations numériques pour construire des modèles non linéaires simples, sans les supposer de type réacto-diffusifs. Il n'y a pas actuellement de piste claire pour aborder la première approche ; en revanche, la deuxième et la troisième devraient permettre d'élaborer une analyse plus intéressante et plus globale de ces phénomènes d'auto-organisation spectaculaires.

\section{Références}

1• S. Benkada et al., « Transport turbulent et autoorganisation dans les plasmas de tokamak", Images de la physique 2003-2004 (CNRS) 24-30.

2•D. Escande, « Plasma thermonucléaire confiné magnétiquement : un système complexe ", Images de la physique 2005 (CNRS) 39-44.

3- G. Belmont et al. « Turbulence dans les plasmas spatiaux », Images de la physique 2007 (CNRS) 29-34.

4 L. Niemeyer et al., "Fractal dimension of dielectric breakdown", Phys. Rev. Lett. 52 (1984) 12.

5• A. Turing, "The Chemical Basis of Morphogenesis“, Philosophical transactions of the Royal Society B, 237 (1952) 37-72.

6• I. Prigogine et R. Lefever, "Symetry breaking instabilities in dissipative systems", J. Chem. Phys. 48 (1968) 1695.

7• V.K. Vanag et I.R. Epstein, "Localized patterns in reaction-diffusion systems”, Chaos 17, (2007) 037110.

8・ H.G. Purwins et al., "Dissipative solitons", Advances in Physics 59, (2010) 485-701.

9• L. Stollenwerk et al., "Measurement and 3D Simulation of Self-Organized Filaments in a Barrier Discharge", Phys. Rev. Lett. 96 (2006) 255001.

10• J. Guikema et al., Phys. Rev. Lett. 85 (2000) 3817. 